\title{
Arguments pour une émancipation de la stylistique structurale de Riffaterre.
}

\author{
Vessah Ngou, Donald \\ MODYCO, UMR 7114 - CNRS Université Paris X \\ dovengou@u-paris10.fr
}

Comme toutes les autres sciences du langage ou les autres sciences sociales, la stylistique a croisé, grâce à Riffaterre (R.), l'époque structuraliste, avec ce que cela comporte comme renforcements théoriques, mais aussi comme problèmes irrésolus. Nombreux sont ainsi les projets structuralistes qui ont souffert, à tort ou à raison, de préjugés défavorables, notamment au sujet de leur essence immanentiste, insensible au sujet, à l'histoire, au réel, au vrai. Aussi Genette (1966: 156) établissait-il, dans son article « Structuralisme et critique littéraire », le constat suivant : "d'une certaine manière, la notion d'analyse structurale peut être considérée comme un simple équivalent de ce que les Américains nomment le close reading et qu'on appellerait en Europe [...] étude immanente des œuvres. » On eût dit qu'une telle remarque était tournée vers le symbole du métissage linguistique et épistémologique que représentait R., dont la stylistique est encore perçue et enseignée aujourd'hui par certains comme principiellement immanente, close et même sclérosée, orientée à la seule linéarité matérielle du texte.

Nous voudrions ici éprouver, à la lumière de travaux d'exégètes du stylisticien et de ses propres œuvres, combien la vision riffaterienne du style, si incomplète et inachevée ${ }^{1}$ qu'elle a pu paraître dans la décennie de son élaboration, était prospective. S'inscrivant dans un structuralisme sophistiqué, l'appareillage méthodologique d'alors posait déjà les bases d'un dessein expansif par principe, susceptible d'être approfondi et ajusté aux évolutions de la discipline, dans une perspective d'épanouissement du texte et de la praxis stylistique. Dès lors, l'émancipation dont il s'agit apparaît moins comme une ouverture de l'esprit originel de la théorie que comme un appel à l'éclatement de ses applications actuelles.

La vérité est que l'approche structurale du style est l'une des plus puissantes méthodes capables de répondre aujourd'hui aux nouvelles questions posées à la stylistique ou par la stylistique à elle-même. Outre le fait incontesté qu'elle a fourni le plus grand effort de théorisation de la pratique stylistique, en arrachant cette dernière aux plates représentations que s'en faisaient les disciplines externes, en lui définissant définitivement un domaine analytique distinct de la linguistique, elle est la plus à même de dépasser et de fédérer les autres disciplines du texte dans l'économie d'une même démarche. Au premier chef de ces disciplines la critique littéraire et la sociologie de la littérature, notamment avec les notions de champ, d'habitus, et même d'historiographie littéraire, qui éclairent des pans tout entiers du style en tant que manière d'être et en tant que monde sémiotique déterminant la production et l'interprétation. C'est à cet horizon que nous voudrions expérimenter l'ouverture de l'approche riffaterrienne, sans en trahir la pensée et en en conservant de fait l'essentiel des concepts opératoires.

Notre propos, bien évidemment, ne sera guère normatif, dans le sens d'une fixation d'horizon structurel ; il n'aura pas non plus la prétention de procéder à des applications inédites en matière de démarche stylistique. Mais il vise simplement à recontextualiser l'apport de R. aux évolutions et exigences actuelles de la discipline, en montrant en quoi il peut être l'appareil de prédilection en faveur de la compréhension et l'approfondissement des objets culturels et sociaux que sont les textes littéraires. Enfin, faut-il le préciser, cet article ne sera pas un panégyrique à l'instigateur de la stylistique structurale. C'est dans cet esprit, d'ailleurs, qu'il convient aussitôt de prendre des distances par rapport à certains aspects de l'arsenal heuristique riffaterrien, avant d'en préciser les modalités d'ouverture. 


\section{Riffaterre et le difficile endiguement du contexte.}

Substituant, à la norme générale, jamais unanime et difficilement appréciable, celle de norme stylistique, R. propose d'abandonner toute nomenclature rhétorique fastidieuse pour identifier le fait de style uniquement dans le texte, seul antre de l'expérience esthétique. Désormais, l'écart n'est plus perçu par rapport à quelque norme linguistique absolue, mais vis-à-vis des linéaments du texte (pattern), aux modèles dans lesquels il installe le lecteur au fur et à mesure que se déploie l'énoncé. C'est en décalage avec ce pattern que le fait de style crée contraste et attire l'attention. Subséquemment, la norme se fait contexte, arrière-plan concret et permanent qui sert de toile de fond et de régulateur pour le procédé, les deux (norme et contexte) formant les polarités de la structure stylistique : "le contexte stylistique est un pattern stylistique rompu par un élément qui est imprévisible, et le contraste résultant de cette interférence est le stimulus stylistique » (« Critères pour l'analyse du style » in R., 1971 : 57).

Après avoir noté que le contexte est variable, R. précise le jeu de saillance qui s'opère entre stylistique et (norme) linguistique : «seule cette variabilité peut expliquer pourquoi une entité linguistique acquiert, modifie ou perd son effet stylistique en fonction de sa position, pourquoi chaque écart à partir de la norme n'est pas nécessairement un fait de style et pourquoi effet de style n'implique pas anormalité » («Le contexte stylistique » in R., 1971: 64). Et il ajoute («Problèmes d'analyse du style littéraire » in R., $1971: 97)$ : « décrire le système d'une parole par rapport à celui de la langue ne suffit pas à appréhender le style, car la parole ne rend pas compte de la structure spéciale des effets [...]. Cette spécificité rend nécessaire de concevoir un système propre au style et qui le différencie de son véhicule linguistique ». Nous voilà prévenus que l'étiquette structurale de la stylistique riffaterrienne ne relève ni d'une terminologie approximative, ni d'un accident de métalangage, encore moins d'un effet de mode. A l'évidence, la spécificité de la structure stylistique est irréductible aux canons du structuralisme langagier : quoique la linguistique fournisse à la stylistique son outillage heuristique de base, elle n'en constitue paradoxalement qu'un aspect. Quoi de plus normal, au demeurant, tant il est clair, comme le relevaient Cressot et James (1988: 15), que le fait stylistique «est d'ordre à la fois linguistique, psychologique et social». Cela n'a pas d'implications uniquement vis-à-vis du locuteur qui intègre ces dimensions afin d'être bien compris, mais aussi et surtout à l'égard de l'analyste.

Suspendons là notre exégèse du théoricien, que d'autres avant nous ont exercée ${ }^{2}$, pour en identifier directement les temps forts dans ce qui est ici notre projet. Le contexte stylistique constitue le point de départ de cette articulation.

R. (1971 : 89), après avoir effectivement admis la flexibilité du contexte, en précisant qu' «il est susceptible d'applications diverses et peut donner lieu à une structure harmonieusement et logiquement hiérarchisée », en distingue alors deux types : « contexte à l'intérieur du procédé stylistique et contexte à l'extérieur du procédé stylistique, c'est-à-dire entre le contexte qui crée l'opposition du procédé stylistique et le contexte qui modifie cette opposition dans le sens soit du renforcement soit de l'affaiblissement» (p. 67). Le premier est le microcontexte et le second, le macrocontexte. Le microcontexte est limité dans l'espace par sa relation au contraste, et le macrocontexte, longueur de texte plus ou moins variable, précède le procédé stylistique et lui est extérieur; il a par ailleurs un rôle de nivellement, pour renforcer, affaiblir ou rendre manifeste le fait de style. La traditionnelle illustration du microcontexte demeure l'oxymore relevée dans «cette obscure clarté qui tombe des étoiles » du Cid, où clarté vient contraster avec le (micro)contexte obscure. Selon la linéarité de la phrase, l'adjectif obscure est anodin dans son occurrence, mais cette banalité est soudainement rompue par sa succession immédiate avec clarté, qui vient frapper l'imagination et ébranler la recevabilité du terme précédent.

C'est là une idée astucieuse que de dégager le procédé stylistique en fonction de quelque rupture par rapport à une toile de fond textuelle. Cependant, une telle ingéniosité ne va pas sans imperfection. Le fait est que R. semble avoir sous-estimé le trait débordant de la notion de contexte, qu'il a tenté de faire tenir, dans une application au poème, à l'environnement graphique du procédé stylistique. Le véritable problème du contexte stylistique repose ainsi, pour l'essentiel, sur le macrocontexte, dont nous convenons avec Hardy (1969 : 93) qu'il s'avère, malgré le qualificatif macro, singulièrement étroit ${ }^{3}$. Franchement, 
s'il fallait que le contexte soit macro, aurait-il suffit qu'il fût éprouvé à l'échelle de l'environnement textuel (de la période, du paragraphe ou, tout au plus, des pages), qui plus est d'un poème ?

En se focalisant sur le repérage du fait stylistique selon un contexte neutre ou marqué, R. paraît oublier de mettre explicitement en valeur les aspects analytique et interprétatif du contexte, même s'il les développe autrement, notamment par l'ouverture laissée aux recontextualisations (nous y reviendrons). Nul n'ignore en effet que le contexte est doté d'au moins trois forces : le contexte comme zone de localité d'une occurrence, le contexte comme situation de communication et le contexte comme ensemble d'éléments qui entrent en compte dans le processus d'élucidation. Or, tel qu'élaborés, le micro et le macro contextes sont considérés selon leur proximité ou éloignement graphique par rapport à l'occurrence ; soit le premier niveau mentionné. D'un autre côté, il est évident que c'est la mémoire textuelle convertie en ces deux types de contextes qui octroie la clé pour le premier pas de l'analyse : l'identification même du phénomène qu'on aura à examiner. Mais R. aurait pu opérer une exotopie du repérage pour aller de l'avant et reconsidérer le contexte dans la plénitude de son envergure, d'autant plus qu'il en manifeste explicitement l'intention dans « Le contexte stylistique ». Il dit ceci (1971:66) : « cette étude, dont le but est essentiellement prospectif, s'efforce de rendre compte aussi simplement et complètement que possible des différents aspects du contexte. » D'autre part, tout en se disant bien avisé des problèmes que pose, dans ses limites, la notion de «texte aujourd'hui au premier plan des recherches stylistiques », il magnifiait particulièrement un trait chez le linguiste suisse : "Saussure's genius was to understand that the text's true centre is outside the text » (R., $1974: 72)$.

Rendu à ce niveau, quelles sont les corollaires des trois potentialités du contexte articulé à la notion connexe de texte ? Dégageons-en succinctement les équivalences et implications, pour un premier temps :

- le contexte comme environnement textuel se rapporte au co-texte. D'une part il crée le stimulus (microcontexte) et est intérieur au procédé stylistique ; d'autre part, lié à une plus grande mémoire, il renforce le stimulus, l'affaiblit ou le neutralise, en même temps qu'il est en dehors du procédé stylistique (macrocontexte) ;

- le contexte comme situation d'énonciation implique de lourdes conséquences relativement à la distance qui sépare le genre poétique du genre romanesque, précisément avec la complexité des strates énonciatives du dernier. Ainsi, cette situation peut être ramenée au contenu narré, notamment dans les impacts des propos et comportements des personnages sur le cours du récit, comme elle peut être articulée au texte tout entier émanant d'un énonciateur situé dans un cadre d'activité particulier. Réside à la base de ce postulat le principe écologique, selon lequel tout texte est produit dans une pratique / situation sociale déterminée ;

- enfin le contexte comme ensemble d'éléments à prendre en compte dans le procédé d'élucidation. C'est celui qui touche le plus à la culture du lecteur, au sens plein du terme. Car l'intertextualité chez $\mathrm{R}$. englobe tant les textes du même genre littéraire que les textes d'autres genres, qui accroissent le capital intellectuel du décodeur et l'aident à résoudre des problèmes d'analyse. Cet angle du contexte touche aussi aux ajustements selon les visées et les degrés d'analyse envisagés.

Sans être séparées, ces essences du contexte sont bien évidemment liées et se produisent dans la chaîne du procès d'élucidation. Il est donc difficile de savoir a priori où commence et s'arrête tel aspect. Toujours est-il que, ainsi conçu, le contexte, et quelle que soit sa perspective, est un outil très peu disponible. Même l'intertexte, qui constitue le pattern de fond extrême, est à construire : "tout rapprochement intertextuel sera réagi, imposé, non par des coïncidences lexicales, mais par une identité structurale, le texte et son intertexte étant des variantes de la même structure » (1979: 132 ; c'est R. lui-même qui souligne). Comme quoi la réalité même du système textuel c'est son inscription dans un tout à bâtir, autant dire une contextualisation.

Qu'il soit bien clair que ces lacunes présumées sur la visibilité du capital contextuel n'invalident en rien la théorie riffaterrienne, dans la mesure où le théoricien, pour autant qu'il n'ait pas mentionné explicitement ces aspects, les développe parallèlement sous d'autres paradigmes. 


\section{Du contexte interne aux recontextualisations : une théorie de la réception}

Le premier contrepoids que R. apporte lui-même (paradoxalement ?) aux limites de sa modulation du contexte réside dans l'attention accordée au lecteur, non pas seulement comme principal garant de la portée stylistique («la statue dépend de celui qui la regarde»), mais surtout pour les possibles réinterprétations du fait stylistique, en dépit de la fixité du texte. Malgré la perception interne du contexte stylistique, surtout le macrocontexte, voilà le texte posé en termes de recontextualisations indéfinies. Traitant de la «Permanence du message », R. (1971 : 37-38) préconise une équivalence proportionnelle entre la structure linguistique stable d'une part, et un système mental socio-historique dynamique, d'autre part: "les patterns proposés au contrôle du décodage ne subissent aucun changement mais le cadre linguistique de référence du décodeur change avec le temps [...]. Ce phénomène a été négligé parce que ses deux faces ont été considérées séparément (permanence du code utilisé dans l'encodage et le contrôle ; non permanence du code utilisé dans le décodage) ; ce qui détruit son essence. »

Cette dialectique, copieusement développée par le stylisticien, nie précisément tout immanentisme. La restitution du fait stylistique n'est interne que dans la mesure où sa localisation et sa délimitation sont saisies du dedans. Quant à l'élucidation, c'est une question d'attentionnalité : «cette interdépendance entre le procédé stylistique et sa perception est, en bref, tellement au cœur du problème qu'à mon avis nous sommes fondés à utiliser cette perception pour localiser les faits stylistiques dans le discours littéraire » («Critères pour l'analyse du style » in R., 1971 : 42).

Dès lors, le stimulus peut être déclenché par un sentiment externe au texte, selon une toile de fond qui relève non plus du simple plan linguistique, encore moins de la simple proximité textuelle avec d'autres éléments, mais d'un système de signes préalablement repérés grâce à l'expérience que le décodeur a engrangée dans ses lectures précédentes. La prévisibilité est rompue aux yeux du lecteur qui s'est constitué, consciemment ou non, un sentiment de norme, un relief stylistique (contexte) relatif à une suite de pratiques. Ce sont des catégories qui «peuvent être suractivées par le fait que le lecteur les a trouvés dans d'autres poèmes » («Vers la définition linguistique du style » in R., 1971: 135). Suivant cette anagnose (ana, avant et gnôsis, connaissance), R. ira même jusqu'à évoquer « une sorte de macrocontexte mental » :

l'attente est basée sur l'expérience que l'on a de certains faits, cette expérience permettant au lecteur de se construire une norme au modèle implicite. Ces faits, dans le cas du macrocontexte, sont des éléments identiques répétés, ou l'accumulation d'éléments analogues. Dès que le lecteur s'aperçoit que ces faits sont comparables, il les déchiffre comme variants d'une même structure, déduit les règles de cette structure (norme particulière au texte) et il réagit au premier variant où un élément est aberrant, c'est-à-dire imprévisible en vertu de ces règles. (« Le contexte stylistique », 1971: 72-73)

Le recours au contexte et, dans une mesure plus large, au lecteur comme principal axe heuristique, apparaît d'autant plus salutaire qu'il permet de rendre compte de phénomènes ardus pour l'analyse traditionnelle du style, en l'occurrence le fait qu'un même événement puisse se doter, dans des contextes différents, de valeurs stylistiques variables, ou encore qu'il puisse produire tantôt un effet stylistique, tantôt aucun, qu'il soit prévisible dans un contexte et déconcertant dans un autre. A cela s'ajoute une solution à la conception discontinue du fait de style, vu qu'un procédé, pour autant qu'il s'impose par saillance, ne manque pas de fonctionner comme contexte pour porter à son tour un autre fait à l'attention. Du coup, les notions embarrassantes pour la stylistique trouvent avec éclat une rationalisation tangible, tant le paramétrage opéré par R. manifeste une nette lucidité sur les points d'ancrage usuels du fameux écart linguistique. Outre le contexte en soi, la source de l'énonciation devient aussi un facteur déterminant, car le fait pour une unité linguistique d'être neutre indépendamment des autres ne signifie en rien qu'elle le restera une fois intégrée à la composition de l'idiolecte d'un auteur, ou à quelque structure socio-historico-littéraire précise. Ajouté à cela le code écrit, qui est susceptible de renouveler un élément linguistique dans le langage oral. Il est en effet évident que la transposition d'éléments du langage quotidien vers l'écrit n'est pas simple, ne serait-ce que parce que les relations qui les organisent dans leur 
nouvel environnement, sans gommer les caractères qui permettent de les décrire selon la grammaire ordinaire, ne sauraient être les mêmes que celles qui les régissent dans le parler.

Ce n'est pas tout. Le fait que le contexte englobe dans le dispositif de R. tant les conditions d'énonciation que les situations d'élucidation refuse toute valeur stylistique permanente ou intrinsèque à quelque unité, toute conception statique du style, ce qui insuffle un certain dynamisme à la discipline, aussi bien dans le temps que dans la démarche. D’abord dans la démarche : la stylistique littéraire se doit de dépasser les simples études grammaticales. Il en découle un mouvement à deux échelons, stylistique à proprement parler, puis métastylistique. Le premier s'intéresse aux effets locaux des faits dans le texte, à l'aune du cosmos présenté. Il doit cependant être complété par un deuxième, qui se situe dans un autre système de relations, celui des fonctionnements discursifs sur les enjeux de production de standards ou régularités stylistiques articulés à des positionnements idéologiques. Il s'agit ici de définir les conditions d'occurrence ainsi que leur logique de distribution et de fréquences, de telle sorte que le fait de style soit saisi non pas comme une pure construction accidentelle, mais comme un objet à la fois individuel et social, une exploitation des possibles langagiers disponibles ou en élaboration dans le champ littéraire. A se stade, les analyses se doivent effectivement d'être

métastylistiques, [et] portent, non sur le fait de style lui-même, mais sur les réactions
qu'il provoque. Au lieu de situer, d'identifier la cause (de nature linguistique), elles
interprètent l'effet (de nature psychologique); au lieu de délimiter une forme unique
et qui ne change pas, elles rendent compte de quelques unes des perceptions possibles
de cette forme, qui varient avec les temps, les cultures, les lecteurs. La description qui
devrait découvrir, dans le fait de langue, le facteur formel qui le rend stylistiquement
actif (et qui lui donne pertinence dans une structure stylistique), devient un exercice
philologique pour confirmer la réaction première, garantir les rationalisations qu'elle
déclenche, bref consacrer l'impressionnisme. [...] En situant les stimuli dans le
discours, on établira de proche en proche une segmentation uniquement stylistique qui
remplacera les catégories préconçues de la terminologie grammaticale. («Problèmes
d'analyse du style littéraire » in R., 1971:110-111)

Sur le plan de l'histoire à présent, la démarche stylistique acquiert en soi une fonction heuristique probante: sur quelque fait que ce soit, elle n'aspire guère au dogmatisme, encore moins à quelque achèvement, mais se propose d'être gardée en mémoire ; elle s'offre pour poser les jalons en vue d'un type de problématisation nouvelle (du côté de l'analyste), ou de réemploi (du côté des écrivains) ${ }^{4}$. A ce stade, il convient de se préoccuper non seulement des effets stylistiques, mais aussi de leur histoire, de leur variation en termes d'intensité, autrement dit de la façon dont ils pourraient s'établir comme éphémères, éternels ou renaissants. D'où l'insistance de R. sur la théorisation de la réception («Critères pour l'analyse du style », $1971: 38-39$ - l'auteur souligne) :

\begin{abstract}
Je pose donc que le point de vue spécifique de la stylistique doit embrasser cette simultanéité de la permanence et du changement. Il doit combiner synchronie et diachronie, ce que la dichotomie entre encodeur et décodeur rend possible. D'un côté, un état de langage qui a conservé parfaitement sa structure et ses patterns stylistiques de contrôle du décodage, figés par l'écriture. De l'autre côté, les différentes actualisations des potentiels du texte réalisées par les générations successives de décodeurs, dans les limites imposées par les patterns du texte et les codes des lecteurs, avec conflit ou non entre les deux. En étudiant ces combinaisons, nous aurons l'histoire de la survie des effets malgré la disparition graduelle du code de référence pour lequel ils étaient prévus. [...] D’un point de vue stylistique, nous pouvons trouver dans la survie des procédés stylistiques en tant qu'unités efficientes, une confirmation expérimentale de l'expressivité mise en valeur par d'autres moyens d'investigation.
\end{abstract}

Voilà la méthode stylistique dotée de substance sémiotique de grande ampleur pour analyser les conformités, les ruptures et les indifférences par rapports aux repères marqués de la langue littéraire. Audelà de sa perspective formelle, la méthode a vocation aussi à définir des principes de comparaison et les conditions de possibilité pour certains phénomènes langagiers. 
Ce parti pris de R. sur des configurations langagières, définies en termes d'effets et de rendements stylistiques par rapport à l'évolution du champ littéraire et à la perception des analystes, entre en résonnance avec le patron stylistique de Gilles Philippe, qui vient comme en compléter la portée par un apport théorique nouveau ${ }^{5}$. Le patron stylistique, en effet, situe les unités stylistiques dans l'optique d'une histoire des pratiques du discours par la recherche préalable d'archétypes. C'est donc à la fois de la permanence et de l'évolution des modèles de perception qu'il témoigne dans la construction du sens :

\begin{abstract}
travailler un ensemble de textes rapprochés par une expérience de lecture sinon identique du moins relativement homogène et par une inscription historico-sociolittéraire commune permet d'aller vers le fait de style local, afin de remonter, par comparaison, vers des patrons stylistiques dont la visée est aussi heuristique. C'est à partir d'eux que peuvent être envisagés le statut et l'état de la langue littéraire, témoin des imaginaires langagiers d'un moment donné. Les patrons stylistiques permettent à la fois de rendre compte de la matérialité langagière des textes d'un corpus (ils s'appuient sur des configurations syntaxiques) et d'aller du côté d'une herméneutique contrôlée : c'est s'intéresser à la manière dont un texte construit du sens - voire du savoir -, davantage qu'aux intentions - prétendues - de l'auteur. (J. Piat, 2005 : 12)
\end{abstract}

Analogue en son principe à l'archive, le second palier du cheminement riffaterrien, et spécialement le patron stylistique qui en est un avatar, en conservant la trace des actes d'hier et leurs cheminements, sert de flambeau au présent. Voilà pourquoi «le déchiffrement des textes romanesques repose [...] en tout premier lieu sur la reconnaissance de tels patrons et exige une compétence interprétative spécifique » (Maingueneau et Philippe, $2002: 367$ ).

On voit que, dans tout le parcours interprétatif qu'il propose, R. met un accent particulier sur l'articulation entre structure linguistique globale, contexte matériel d'une occurrence langagière de quelque nature qu'elle soit et domaine discursif littéraire, celui des effets de valeur. Dès lors, l'idée que la question du style engendre une analyse des effets du texte à la lecture plutôt qu'à sa production explique pourquoi et comment des éléments du discours qui sont sans contenu de signification à l'émission en sont investis à la réception. La contextualisation prend alors tout son sens de mise en relation d'un texte avec un ensemble de problématiques et de perspectives. En tout état de cause, en plus du domaine linguistique qui demeure fondamental dans la mesure où c'est lui qui procure au texte son matériau,

\begin{abstract}
le procès de poétisation riffaterrien dans lequel intervient l'opération herméneutique entraîne alors pour le lecteur la mise en action d'un processus interprétatif dont l'origine est codée dans le texte mais dont la résolution est située hors du texte matériel donné et qui nécessite une intervention "extérieure » dans deux univers interprétatifs distincts. L'infratextuel est servi par l'hypogramme (pris comme catégorie générale pour le sub-texte, la phrase matrice, etc.) et l'intertextuel est servi par l'interprétant qui convoque dans le processus d'élucidation le savoir culturel (littéraire) nécessaire. (Thomas, $2002: 27$ - les italiques sont de l'auteur)
\end{abstract}

\title{
3 Etude de cas : quelques pistes de réception pour une micro-unité de néologie lexicale
}

Suite à la mise en relief de l'assise méthodologique sur les plans généraux du contexte et des contextualisations, nous pouvons à présent procéder à une mise en pratique de cet outillage pour le ramener spécifiquement au contexte stylistique riffaterrien.

Pour ce faire, nous réaliserons une analyse concrète afin d'éprouver de façon extrême comment, de fil en aiguille, des données linguistiques apparemment anodines se dotent d'effets stylistiques dans un contexte de fond, selon la culture du lecteur. Le stimulus est approfondi suivant le discours dans sa spécificité fonctionnelle, dans un panorama intertextuel, transtextuel. A cette étape suprême, «la référentialité est suspendue parce que les marques, le non-dit du texte, ne peuvent être complétés et expliqués que par un intertexte » (R., $1994: 289)$. 
Pour des besoins de clarté, nous suivrons une progression du traditionnel écart linguistique (si tant est que cela soit légitime) à la vision du fait stylistique par R. dans sa plénitude. En nous appuyant sur cet extrait de L'Aîné des orphelins de Tierno Monénembo (2000:21), roman inspiré du génocide rwandais, partons de l'unité minimale de créativité linguistique qu'est la dérivation :

$$
\begin{aligned}
& \text { «... c'est là qu'on a entassé les leaders, les proxénètes, les auteurs de parricide et les } \\
& \text { génocideurs dont l'âge court de sept à dix-sept ans. " }
\end{aligned}
$$

Dans la perspective riffaterrienne, rappelons que le microcontexte est l'élément du texte par rapport auquel un élément inattendu, le stimulus, forme contraste. Appliqué à la dérivation par suffixation, le microcontexte se restreint à la base (génocid) à laquelle est adjoint le suffixe (eur). Quant à ce dernier, il représente alors le stimulus. Nul ne contestera en effet que le mot non marqué pour désigner des auteurs de génocide est bien génocidaire, prévu par le dictionnaire à la fois comme nom et adjectif pour référer à ce qui est relatif au génocide ou bien qui y prend part. Cependant, Monénembo propose une entrée spéciale qui, remplaçant le suffixe d'adjectif aire insuffisamment expressif, expliciterait l'idée d'actant, voire de profession liée à l'activité du génocide.

Mais ce qui sera intéressant c'est de suivre les phases où ce stimulus est apte à constituer une brisure d'équivalence: après le panorama linguistique pur, intervient une activité de lecture qui implique subséquemment la reconnaissance d'une forte présence d'éléments saillants et communs à un ensemble de textes issus du même univers langagier. C'est que les mots ne signifient plus

$$
\begin{aligned}
& \text { par référence à des choses ou à des concepts, ou plus généralement par référence à un } \\
& \text { univers non-verbal. Ils signifient par référence à des complexes de représentations } \\
& \text { déjà entièrement intégrés à l'univers langagier. Ces complexes peuvent être des textes } \\
& \text { connus, ou des fragments de textes qui survivent à la séparation de leur contexte, et } \\
& \text { dont on reconnaît, dans un nouveau contexte, qu'ils lui préexistaient. Ces complexes } \\
& \text { finissent par devenir la monnaie courante du sociolecte. [...] Ainsi compris, la } \\
& \text { production du sens dans l'œuvre littéraire résulte [aussi] de la connaissance du mot } \\
& \text { comme membre d'un ensemble... (R., 1981:6-l'auteur souligne) }
\end{aligned}
$$

Il y a là de quoi faire penser au concept de convergence, dont R. garantit qu'il peut fonctionner comme contexte, et qu'il emploie en référence à l'accumulation de plusieurs procédés stylistiques indépendants pour produire un faisceau de significations : «seul, chacun serait expressif en soi. Ensemble, chaque procédé stylistique ajoute son expressivité à celle des autres. En général, les effets de ces procédés stylistiques convergent, en un soulignement particulièrement frappant ». Reconnue comme étant la forme stylistique la plus complexe, la convergence a le double avantage d'abaisser, de par sa nature cumulative, le seuil de perceptibilité du procédé stylistique et d'en démontrer subséquemment la réalité physique. Elle pallie les cas où le contexte graphique précédent ne permettrait pas la sensation du contraste. Enfin, c'est un procédé décisif pour contribuer à éclairer avec crédibilité des modalités d'authenticité du concept choix : «même si elle s'est d'abord formée inconsciemment ou si elle est fortuite, elle ne peut échapper aux yeux de l'auteur quand il relit. Qu'elle soit simplement maintenue ou élaborée, elle représente un exemple de conscience extrême de l'utilisation du langage. » («Critères pour l'analyse du style », pp. 6062).

Qu'il nous soit permis à présent de poser le tableau ci-après qui exemplifiera, de par son quadrillage, « la manière de voir [les occurrences], de les comparer, de les interpréter par conséquent dans leur inséparabilité même » (R, $1980: 15)$. L'on excusera aussi l'opulence des exemples, attendu que la preuve du caractéristique lié au style exige que la récurrence compte de la même façon que l'occurrence.

Ce qui légitime ici notre démarche, autant que la convocation de ces extraits, c'est rappelons-le, l'essence même du macrocontexte. Insistons-y donc :

son effet (du macrocontexte) sur le procédé stylistique est reconnu grâce aux réactions du lecteur, le point de départ de cette perception du contexte pourrait être conçu comme variant suivant l'attention et la mémoire du lecteur, c'est-à-dire sa capacité à reconnaitre des similarités et des disimilarités formelles [...] (dans ce cas une forme donnée est facile à remarquer et surprenante parce qu'elle est récurrente dans un 


\begin{abstract}
contexte donné - sémantique ou situationnel - contexte dans lequel son seul conditionnement est d'ordre psychologique; dans ce cas le lecteur se rappelle l'avoir déjà vu en dépit du fait que l'espace textuel qui le sépare de la dernière occurrence est d'une longueur et d'une variété qui excèdent de loin ce que l'on pourrait raisonnablement attribuer au contexte en tant qu'unité fonctionnelle). («Le contexte stylistique » p. 81)
\end{abstract}

Tableau ${ }^{6}$ :

\begin{tabular}{|c|c|c|}
\hline $\mathrm{N}^{\circ}$ & Prototypes et co(n)textes & Références \\
\hline 1. & Le compteur enregistreur des fusillés marquait entre quatre et cinq cents par jour... & L.V.D., 29 \\
\hline 2. & $\begin{array}{l}\text { - Le juge m'a dit qu'il y a trois catégories de complices : les complices (de zéro à cinq ans), les } \\
\text { exécutants (de cinq à vingt ans) et les organisateurs (la perpétuité ou la potence). }\end{array}$ & A.O., 87 \\
\hline 3. & Yavelde était assez jolie fille pour ne pas manquer de vengeurs. & A.P., 72 \\
\hline 4. & - Je veux que dans le temps de poireauteur on prenne le temps de vivre et de rêver. & A.P., 107 \\
\hline 5. & $\begin{array}{l}\text { La rue la présentait (Yealdara) comme une proie facile. Alors, on était combien à frotter comme lui ? } \\
\text { Et qui était le meilleur cogneur du tas? Le bon frotteur? Celui qui frotte juste. Qui remuait les buts } \\
\text { comme on dit dans ce pays? Qui était le maître cogneur? }\end{array}$ & A.P., 119 \\
\hline 6. & $\begin{array}{l}\text { [Le guide] fit construire à tous les coins de rues des «regardoirs » de cuisses droites, toujours } \\
\text { accouplés : un pour hommes, et un pour femmes, sous prétexte qu'on regardait jour et nuit, certains } \\
\text { "regardeurs » mirent des lits, d'autres se contentaient des stations debout ou des sols. Neuf mois après } \\
\text { l'installation des regardoirs, le pays connut un boom de population... }\end{array}$ & L.V.D., 132 \\
\hline 7. & Salimata implorait Allah, l'absoluteur et le miséricordieux, pour que passât [chez elle] la maternité. & S.I., 44 \\
\hline 8. & $\begin{array}{l}\text { La clameur appela d'autres pleureuses et lamentations et se communiqua à tout le village. [...] La cour } \\
\text { était toute jonchée de pleureuses, assiégée par une légion de curieux, [puis] le vacarme s'arrêta... }\end{array}$ & S.I., 104 \\
\hline 9. & Le griot Diamourou se plaça à droite [...] et on attendit les vagues de salueurs. & S.I., $106-107$ \\
\hline 10. & $\begin{array}{l}\text { Les derniers salueurs étaient repartis. Tout le monde se moquait de [Balla]. Un féticheur, un lanceur } \\
\text { de mauvais sorts. }\end{array}$ & S.I., 111 \\
\hline 11. & Djigui [aimait] se faire célébrer par les adulateurs et les griots... & M.O.D., 15 \\
\hline 12. & Suivi de mes séides et louangeurs, nous arrivâmes au Kebi... & M.O.D., 76 \\
\hline 13. & On cessa de nous envoyer les collecteurs et les recruteurs. & M.O.D., 202 \\
\hline 14. & Mais des excités, des provocateurs professionnels tirèrent sur les forces de l'ordre. & M.O.D., 273 \\
\hline 15. & «Toi, je connais. Tu es avant à Abidjan, transporteur, multiplicateur de billets, guérisseur et tout. » & A.P.O., 126 \\
\hline 16. & [Le colosse prit] ses lunettes de révolutionnaire croqueur de gamines au lycée Karl-Marx. & $Y . V ., 20$ \\
\hline 17. & $\begin{array}{l}\text { [Ils] avaient formé des délégations : tous les beaux parleurs de Côte, les négociateurs chevronnés, les } \\
\text { palabreurs de carrière ou de fortune, les argumentateurs-nés, les désamorceurs d'embûches. }\end{array}$ & S.S.L.L., 84 \\
\hline 18. & [Arthur] invitait vedette sur vedette, tombeur de femmes sur tombeur de femmes. & S.S.L.L., 113 \\
\hline 19. & les aboyeurs de Nsanga-Norda octroyaient [à notre ancien président] une pincée de gloire séculaire... & S.S.L.L., 132 \\
\hline 20. & Ce que le raconteur Machelo Palma ne pouvait pas dire c'est la nature de la petite bête... & S.S.L.L., 173 \\
\hline 21. & « Méprise avec force cette calomnie, et surtout garde-toi d'écouter le « mauvais suggestionneur »... & O.M.C. 131 \\
\hline 22. & $\begin{array}{l}\text { On lui envoya aussitôt des poignadeurs et des donneurs de poison, des étrangleurs et des dresseurs } \\
\text { d'abeille. }\end{array}$ & $\begin{array}{l}\text { R.K. } \\
228\end{array}$ \\
\hline
\end{tabular}




\begin{tabular}{|r|l|l|}
\hline 23. & les pleureuses, la chorale, la mine éplorée des proches, tout cela assurerait un spectacle... & C.J.V., 16 \\
\hline 24. & L'interprète conseilla à Keita d'affecter, volontairement, des éventeurs aux gradés noirs... & M.O.D., 54 \\
\hline 25. & $\begin{array}{l}\text { [Le colosse] était le libérateur qui devait donner une bonne leçon aux monopoleurs, aux trafiquants, } \\
\text { aux mystificateurs de la cause du peuple. « Il fera une bouchée de tous nos tordus magouilleurs ! » }\end{array}$ & Y.V., 59 \\
\hline 26. & [La Badiène] avait été la marieuse de « sa fille ». & $X ., 15$ \\
\hline
\end{tabular}

Au sein de [6], l'un des narrateurs nous présente l'historique d'une notion, celle de regardoir, dont la forme est constituée du radical regard et du suffixe -oir, relatif au lieu où se fait l'action, à l'industrie ou bien au produit de l'industrie. Les regardoirs de cuisses droites sont des locaux spécialement conçus par le guide à travers tout le pays pour scruter les cuisses droites des femmes, afin de retrouver Chaïdana, l'amante du dictateur, sur le membre de laquelle il avait lui-même inscrit une marque. C'est dans les regardoirs qu'opèrent les regardeurs de cuisses droites.

Est-il besoin d'analyser chacune de ces occurrences qui, signalons-le, pullulent dans les textes ${ }^{7}$ ? Un détour simplement pour relever un effet remarquable : vu le foisonnement du suffixe dans les énoncés, même un mot banal comme auteur (cf. l'exemple initial hors du tableau) pourrait susciter un renouvellement vigoureux du terme par une remise en valeur du sens du suffixe. On voit ainsi, comme prédit par R., comment une unité linguistique neutre acquiert un effet stylistique en fonction de sa position: quoique auteur ne renferme pas de fonction suffixale pour la graphie -eur, il est susceptible d'évoquer fortement, dans le cadre général du pattern contextuel qui en prépare les effets, non plus le contenu de performateur, mais celui de professionnel pour commettre des parricides. C'est un résultat similaire qui s'observe avec organisateurs dans [2], vengeurs de [3], poireauteur en [4], (maître) cogneur puis frotteur de [5], collecteurs et recruteurs de [13], puis transporteur de [15], qui saisissent un sémantisme profond lié à une occupation régulière (relative respectivement à la planification des génocides, à la rancune, à l'attente, celle d'une plénitude existentielle, à des activités libidineuses à souhait, à la saisie abusive de biens privés, à l'enrôlement, enfin, à l'expédition d'argent), et non plus à un exercice ponctuel.

Il en va de même avec poignadeurs, donneurs de poison, étrangleurs et dresseurs d'abeilles de [22], qui modalisent épistémiquement les activités décrites dans le sens de véritables professions. Syntaxiquement rapprochés de l'expression usuelle dresseur d'abeilles (comme dresseur de chiens, dresseur de lions, etc.) les autres modèles éveillent l'attention sur le suffixe et le dotent énergiquement du sémantisme professionnel dans cette dernière formule. À l'opposé, l'expression usuelle conquiert également un trait inaccoutumé et prend une teneur stylistique subtile. On aboutit à la présentation d'une société où les activités les plus meurtrières cohabitent sans scandale avec les métiers les plus usuels. Pareillement, en [1], quoique l'on puisse objecter que compteur existe déjà en langue, il saisit dans sa collocation, voire sa polylexicalité, avec enregistreur des fusillés un trait inhabituel, et l'ensemble ferait penser à un appareil spécialement conçu pour calculer la quantité des victimes de l'oppression du régime en place.

Une fois la ressemblance de ces faits établie, nous pouvons désormais tenter de «déduire les règles de cette structure » qui combine une signifiance textuelle (dans l'univers du roman) et socio-littéraire (dans le cadre de vie des auteurs), et qui contribue à éclairer les paliers qui structurent le style selon R. Le premier axe, qui n'exclut pas le second, pourrait se formuler ainsi : concrètement, l'ensemble des mots produits accentue la force numérique des éléments référés et signifie un penchant pour l'excès. Globalement, il se dessine, au niveau de l'effort référentiel, une organisation des sociétés mises en scène selon des activités banales, pour ne pas dire selon une oisiveté attestée. L'inactivité générale fait qu'il y ait parmi les populations des spécialités insolites : salueurs (du matin / du soir), causeurs, insulteurs, pleureuses, prieurs, chasseurs, creuseurs, mangeurs (de doubles), palabreurs, demandeurs, etc. Les tâches ne sont en rien rémunératrices au point de faire vivre financièrement leurs adeptes, néanmoins sur le plan occupationnel, c'est d'elles qu'ils vivent.

Mais saurions-nous arrêter là l'interprétation, comme si cette accumulation d'occurrences pourtant indépendantes était fortuite? Les prototypes ainsi soulignés et une fois rassemblés se dotent d'une 
consistance imposante. Partant, il ne serait pas précipité de dire à cette étape d'analyse que le fonctionnement du suffixe -eur dans ce contexte régulateur est analogue en son principe à une forme conventionnelle, concept qui établit à lui seul une réflexion linguistique sur les rapports entre universalité et particularités culturelles, géographiques, esthétiques. Tout en soulignant la cohérence d'un style, il lui assure sa valeur de modèle à la fois collectif et singulier. La forme conventionnelle est
tout fait de style perçu également comme caractéristique d'une tradition, ou d'une doctrine esthétique, ou d'une langue spéciale. Le fait de style ordinaire ne dépend que du contexte, qui le crée toujours, parfois l'active, parfois le neutralise. La forme conventionnelle dépend aussi du contexte, mais elle tient de ses origines une valeur intrinsèque qui ne peut être annulée entièrement [...]. Aussi pose-t-elle des problèmes ardus au critique : il n'en peut démontrer le mécanisme stylistique sans recourir à des faits extralinguistiques, puisque par définition la convention est une règle imposée du dehors à la structure verbale. («L'étude stylistique des formes littéraires conventionnelles » in $1971: 182-183)$.

Appliquée à nos occurrences, cette «valeur intrinsèque imposée du dehors à la structure verbale » pourrait, à ce point transitoire, s'exprimer comme suit : du point de vue purement nominatif, il se produit fortement dans l'imaginaire africain un caractère singulier de la vision du monde, en vertu duquel les êtres sont classés selon les pratiques qui les occupent à longueur de journée, et les choses selon leurs fonctions d'usage. Sur le plan stylistique, ce procédé fonctionne bien sûr comme un artifice langagier dont les enjeux sont rentables, mais surtout, il apparaît sous le prisme d'une matrice de représentations mentales inscrites dans des catégories nominales ajustées. L'on assiste à un mode de substitution lexicale

\begin{abstract}
pour résoudre un problème précis de nomination: il s'agit de traduire le discours taxinomique africain qui consiste à organiser l'espace social et culturel par référence soit aux activités professionnelles des acteurs (les forgerons, les tisserands, les cultivateurs) soit aux habitudes et attitudes des gens («les assis », « les sollicités », «les décédés », «les enceintes », « les naissances »), etc. Le mode d'être se substitue dans la nomination. Ici, plus qu'ailleurs, a lieu cet exercice de traduction d'une vision du monde, en l'occurrence d'une certaine « africanité » du dire qui se loge, non dans une substance précise à nommer, mais dans un imaginaire, lequel est, comme on le sait, avant tout virtualité, c'est-à-dire processus abstrait. (Kouassi, 2007 : 131-132)
\end{abstract}

Soyons plus concret dans la rationalisation de ces effets qui convergent vers le principe de cohésion mentionné supra. Concernant les noms de métiers, il est évident qu'ils ne sont pas forcément générés dans la majorité des systèmes primaires, dont font partie les langues africaines, selon un principe analogue au français. Des tournures périphrastiques seules aident à nommer certaines occupations ou outils, traditionnels et non traditionnels, lorsque l'emprunt ou d'autres facteurs externes d'évolution linguistique ne procurent pas de dénomination. Dans une langue camerounaise par exemple, le Shu pamom $^{8}$, le seul morphème préfixal nga ([nga]) suffit devant un verbe à produire un substantif qui rend parfaitement compte du métier ou de l'attitude exprimée par le verbe. Quant aux dénominations d'appareils-instruments, le préfixe you remplit ce rôle. Ainsi, l'architecte sera appelé nga kut ndap (littéralement celui qui construit la maison), le maçon nga yapshe ndap (celui qui dispose la maison), le chauffeur nga su matua (celui qui conduit le véhicule), le décapsuleur you sho' nzu (ce avec quoi on ouvre la bouteille), le cric you kyem matua (ce avec quoi on soulève la voiture), etc.

Précisons bien que c'est moins le morphème d'origine ou quelque autre unité lexicale, que la tendance à la nomination, qui est transposé en français. La pratique du récit, justement, constitue un champ d'observation approprié en faveur de cette disposition discursive, notamment dans les modalités de cohésion au sein de la chaîne narrative. Au cours du récit oral en effet, la seule évocation d'une personne qui aurait produit un acte, même ponctuel, suffit, dans la suite cotextuelle de la narration, à cristalliser son action dans une nomination telle que nous venons de le voir. Cette série d'occurrences empruntées à un auteur dit de la première génération, Kourouma, aident à illustrer ce procédé davantage :

[27] «Il fallait honnir [Bamba], l'empoigner, le mordre. Et Fama avança sur l'insulteur. » (S.I., 16) 
[28] « On chuchote que Balla brûlera les fétiches, se convertira et se courbera, insinua un causeur.» (S.I., 111)

[29] «Le chef du clan $d u$ déserteur recevra vingt coups de fouet et un des frères $d u$ déserteur sera requis. Que le déserteur soit sans frère, son chef de clan sera puni...» (M.O.D., 62)

[30] « Un mois, il a été [...] emmerdé par les palabreurs... » (A.P.O., 40)

[31] «Le frappeur de tam-tam avança vers elle, se courba à ses pieds et joua en son honneur. » (A.P.O., 125)

Dans le prototype initial, le cotexte de gauche met en scène Fama dans une querelle avec Bamba. Ce dernier vient effectivement d'insulter Fama, qui perçoit les paroles comme un affront suprême à sa dignité de prince. Le mot insulteur ne renvoie pas à proprement parler à une occupation, mais sert à rappeler à propos l'action qui a causé la colère du héros. C'est un procédé courant dans les récits oraux africains, que d'évoquer à nouveau le sujet par une nomination qui identifie son action à lui-même. De telle sorte que, en se situant dans l'optique de l'hypotexte dont procède le français, si un auditeur qui n'a pas bien suivi le fil du récit, pour comprendre qui est l'objet des empoignades et morsures, demandait mordre qui ?, le conteur lui répondrait, sans que ceci soit perçu comme une exagération ou un écart de langage, celui qui insulte (l'insulteur). Le présent, dans celui qui insulte, produit à l'égard de l'hypotexte l'effet d'une action initialement captée dans sa ponctualité, mais reproduite dans une virtualité indéfinie. Il en va de même avec causeur en [28], qui n'est doté de pertinence que dans la mesure où il évoque dans l'indétermination une personne parmi tant d'autres qui murmurent au sujet de Balla. Pareil avec [29], qui se situe à la suite d'une mise en garde des envahisseurs du village contre toute tentative de désertion.

Ces exemples sont convoqués à dessein pour tracer les courbes du processus narratologique qui se prolonge et s'intensifie de façon particulière dans le récit traditionnel. Cela n'est d'ailleurs pas un hasard si les extraits émanent d'un auteur comme Kourouma, qui n'a cessé de marteler, comme chacun sait, sa détermination à transposer en français le rythme dit africain dans ses romans. L'on aboutit, chez Kourouma comme chez d'autres artistes de sa génération, à une floraison lexicale infinie, où sont simultanément cristallisés dans la nomination l'action, ses actants (sujets et objets) et même les circonstants, pourvu que le schéma attributif selon le rôle ou l'attitude du sujet nommé soit prioritaire. On a ainsi des oublieurs, et pas n'importe lesquels, des oublieurs de locomotion (Y.V., 76), des profiteurs de la fatigue des chiffres, qui sont les bénéficiaires de détournements de deniers publics (L.V.D., 177), des récolteurs d'hommes, êtres essentiellement féminins à vocation séductrice (A.P., 40), des pondeurs de philosophies (L.V.D., 88), des mangeurs d'âmes (A.P.O., 134), des serreurs de mains (Y.V., 129), des tueurs de femelle (S.S.L.L., 99), des mangeurs de viande contre mangeurs de chevaine, des buveurs d'eau de marigots contre buveurs d'eau-de-fer (S.S.L.L., 117), des crieurs de vêpres (S.S.L.L., 119), des penseurs occasionnels (S.S.L.L., 124), des donneurs originels de poux (S.S.L.L., 154), des coureurs d'occasion, semeurs de flotte (S.S.L.L., 190), des bagarreurs du dimanche (O.M.C., 217), des dévoreuses d'hommes $(X ., 61)$. Et que dire de porteurs de chapelets à onze grains (M.O.D., 150), qui laisse ouverte la possibilité d'envisager des porteurs de chapelets à chaque nombre de grains?

Jusqu'ici, nous avons fait comme si le choix des treize textes soumis à analyse était fortuit, comme s'il reposait à la lettre sur la mémoire et l'attention du lecteur prévues par R. Or il est évident que le morphème -eur, et plus encore l'ancrage psycho-socio-littéraire dont il est l'indice ${ }^{9}$, justifie et problématise le statut du corpus qui le porte à notre attention, ne serait-ce que par les règles de prédictibilité que son dépouillement laisse entrevoir. Nous voilà conduits à un cran supérieur du projet riffaterrien. Aussi convient-il à présent de dire un mot sur les critères qui ont présidé au choix du corpus, après quoi s'imposera un bref commentaire sur son unité.

Il s'agit d'abord de la valeur stylistique des romans, souvent soulignée par la critique, puis de notre propre connaissance des textes. Nous référant à l'historiographie littéraire, nous avons, une fois l'éveil sur le morphème suscité, adopté l'amplitude diachronique la plus large possible (1968-2008), de façon à considérer des œuvres d'auteurs de la première génération et celles d'auteurs jeunes. Ainsi, si l'on se situe dans l'optique de la réception des romans, le corpus apparaît pertinent dans la mesure où les romans de Kourouma, Sony Labou Tansi, Hampâté Bâ, Ousmane et même Monénembo s'inscrivent dans une étape spécifique de l'histoire littéraire, celle qui inaugure la période dite des indépendances, par un 
renouvellement de la pratique littéraire et langagière. La subversion langagière, qui jouit à cet instant d'une faveur exceptionnelle, y est également attestée comme un puissant indice de littérarité. Non seulement ce sont des auteurs qui sont encore vivement imprégnés de leur imaginaire linguistique et traditionnel premier, mais ils témoignent d'une hardiesse dans la pratique littéraire. Ceci expliquerait la quasi absence, dans le corpus, d'auteurs jeunes comme Léonora Miano par exemple, qui a quitté son Cameroun natal assez tôt pour s'installer en France. Enfant de la postcolonie qui n'a pas un ancrage profond avec la tradition, elle serait tournée vers des stratégies individuelles plutôt que des appartenances.

On voit ainsi que, en servant de comparant entre les éléments du corpus et ceux qui n'ont pu y être intégrés (on n'est plus porté à vérifier une hypothèse en écartant ce qui semble l'exclure ou la contredire), le seul morphème -eur nous conduit vers un cadre de l'historicité littéraire probant, en même temps qu'il en dit long sur les moments de l'imaginaire langagier et leurs champs de possibles : « la stylistique se fait alors moins analyse d'un texte ou d'un auteur que d'un moment. Et le corpus sur lequel elle se fonde nécessairement - parce qu'elle s'intéresse aux formes langagières mises en œuvre dans les textes littéraires - se transforme ainsi en étalon» (Piat, 2007: 3), afin de bien décoder les points d'enrichissements provenant de nouvelles possibilités stylistiques et les générant en retour.

A l'évocation des auteurs que nous venons de mentionner, d'aucuns chercheraient à vérifier s'il ne s'agit pas là de phénomènes idiolectaux, propres aux seuls écrivains cités, plutôt que de sociolectes relevant de groupements stylistiques irrévocables. Sans nier les nuances d'emplois (le cas de Labou Tansi, par exemple, est particulièrement prodigieux qui met le suffixe au profit de l'expression de débordements comportementaux et sociaux inconcevables), disons d'abord qu'il y va du principe du corpus: les récurrences mises à jour sont virtuellement récursives, ce qui implique qu'une analyse restreinte à un certain nombre de faits ou d'auteurs est susceptible de rendre compte de l'ensemble. Ensuite, la perception idiolectale, sans être hors de propos, nous prive du bénéfice de l'observation in situ, qui ignore les rapports de proportions. R. («Problèmes d'analyse du style littéraire », 1971 : 103) sera même conduit à l'extrême conclusion selon laquelle la langue d'auteur est une entité inutile ${ }^{10}$. Enfin, l'orientation qui est la nôtre n'est qu'une optique attenante au champ littéraire, et qui fait jaillir en permanence la prémisse selon laquelle un style n'est pas fait d'un cumul de traits dépourvus de liens les uns avec les autres. Tous ses traits caractéristiques ont une cohérence et créent une physionomie d'ensemble, qui ne saurait même pas se lire en un assemblage d'idiolectes ${ }^{11}$.

R., disions-nous, définissait les convergences stylistiques comme «l'accumulation en un point donné de plusieurs procédés stylistiques indépendants ». Ce point donné, qu'il revient à chaque fois de définir, peut révéler un système à double face, verbale et non verbale, graphique et non graphique, imposé par des groupements stylistiques. Signalons à ce sujet que l'indépendance des occurrences convergentes telle que soulignée est précieuse pour le contexte stylistique, au moins parce qu'elle suppose que les mêmes faits observés chez différents auteurs échappent à toute concertation préalable, à toute mise en question et s'intègrent dans des schémas d'action et de perception intériorisés par les énonciateurs. En effet, «la pensée du contexte n'est pas une pensée de la cause mais des conditions » (Rastier, 1998:110). Ces dernières mêlent le récepteur avec l'encodeur, en les situant dans un même univers symbolique.

Dans cette perspective, Vandendorpe (1991: 10) ne concevait-il pas le contexte comme "une réalité mentale déterminée par l'activité pensante d'un sujet placé en situation de réception ou de production d'un message »? On croirait entendre Bourdieu, notamment avec sa notion structurelle de champ, élaborée pour rendre compte de la production d'éléments matériels thématiquement et fonctionnellement analogues. Elle est de ce fait inséparable du concept d'habitus, qui rejoint pleinement le style et moule naturellement l'action pensante des agents :

une des fonctions de l'habitus est de rendre compte de l'unité de style qui unit les pratiques et les biens singuliers d'un agent singulier ou d'une classe d'agents. [...] L'habitus est ce principe générateur et unificateur qui retraduit les caractéristiques intrinsèques et relationnelles d'une position en style de vie unitaire, c'est-à-dire en ensemble unitaire de choix de personnes, de biens, de pratiques. Comme les positions dont ils sont les produits, les habitus sont différenciés; mais ils sont aussi différenciants. Distincts, distingués, ils sont aussi des opérateurs de distinctions [...]. 
Les habitus sont aussi des schèmes classificatoires, des principes de classement, des principes de vision et de division, des goûts différents. (Bourdieu, $1994: 23$ )

Les termes de cette définition nous sont familiers, tant on est en pleine phase d'élaboration de cette structure intertextuelle qui situe le texte dans une pratique sociale singulière. Il s'agit du contexte le plus immédiat dont émane l'œuvre. Suivons encore Bourdieu (1980 : 88-89) :

\begin{abstract}
Les conditionnements associés à une classe particulière de conditions d'existence produisent des habitus, systèmes de disposition durables et transposables, structures structurées disposées à fonctionner comme structures structurantes, c'est-à-dire en tant que principes générateurs et organisateurs de pratiques et de représentations qui peuvent être objectivement adaptées à leur but sans supposer la visée consciente de fins et la maîtrise expresse des opérations nécessaires pour les atteindre, objectivement réglées et régulières sans être en rien le produit de l'obéissance à des règles, et, à tout cela, collectivement orchestrées sans être le produit de l'action organisatrice d'un chef d'orchestre.
\end{abstract}

De fait, les patterns linguistiques de l'auteur deviennent à même d'éclairer l'ensemble du champ dans lequel son œuvre est tissée puis vouée à être reçue, et réciproquement. Surtout, l'ordre du langage se voit doublé d'une organicité supplémentaire, touchant aux coordonnées énonciatives du sujet saisi dans son entour social. On en vient ainsi à intégrer ce plus qui fait que le discours devienne irréductible à la langue et à la parole.

Il ne nous échappe pas que, telle qu'émancipée, la démarche semble maintenant enfermée dans un décryptage existentiel, propre à rendre compte du vécu intime des formes et de leurs significations. D'autant plus qu'on sait combien l'évocation de cette dimension culturelle dans les textes est parfois mal comprise (surtout dans le champ littéraire francophone) par certaines sensibilités modernistes, tellement elle éveille le sentiment d'une vision patrimoniale de la littérature. Et pourtant c'est l'appréhension des textes complexes qui s'en trouve facilitée, de manière à éclairer la lecture et minimiser de fait les écueils d'interprétation $^{12}$. D'ailleurs, il faut se rendre à l'évidence que la pensée riffaterrienne est fortement imprégnée de pareille teneur ethnologique. D'où l'exaltation de Thomas (2002: 21-22): «si le terme herméneutique n'avait pas existé dans sa définition laïque, il aurait fallu l'inventer pour Riffaterre car c'est exactement ce qu'il fait : «Herméneutique : théorie de l'interprétation des signes comme éléments symboliques d'une culture ».

Au demeurant, de tels centrages sociohistoriques, et même culturels, sont au cœur du statut du style :

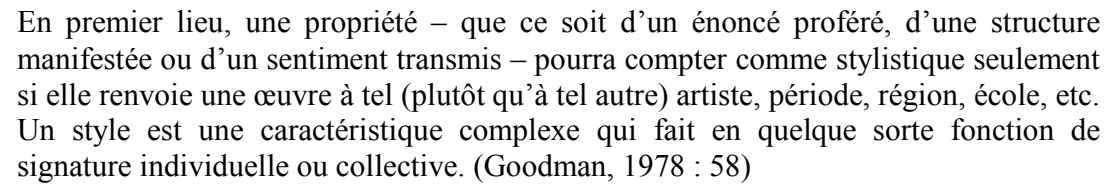

Si l'on part donc du principe que les romans lus, une fois rapprochés dans une structure intertextuelle, sont indexés sur des conditions de production d'un effet de réel relatif à un système socioculturel ou géographique, alors cette situation, toujours particulière parce que tissée selon des traits propres, fait principiellement du cadre historique dont émerge l'œuvre une organicité contexte-norme. Car elle impose sa logique propre et ses dépendances internes autant qu'elle tisse un réseau d'éléments relativement suffisants, saisis dans une esthétique holiste. Aussi les attributions contribuent-elles largement à la compréhension des œuvres en tant qu'artistiques :

la connaissance de l'origine de l'œuvre, même si elle résulte de l'analyse chimique ou d'autres moyens purement scientifiques, informe sur la façon dont le travail doit être regardé, écouté ou lu, en fournissant une base pour découvrir les moyens non patents par lesquels l'œuvre diffère de ou ressemble à d'autres œuvres. [...] Situer une œuvre est esthétiquement significatif en soi, dans la mesure où cela permet de découvrir des qualités comme celles du style. [...] pour autant que l'esthétique est concernée, l'attribution constitue un préliminaire ou un auxiliaire à [sic], ou un sous-produit de la perception du style. (Goodman, $1978: 64$ ) 
C'est seulement à ce prix qu'on échappe au risque de passer à côté de ces faits qu'on «ne peut omettre sans mutiler le texte et [qu'on] ne peut déchiffrer sans les trouver significatifs et caractéristiques » ( «Critères pour l'analyse du style », p. 31). Voilà, finalement, en quoi aussi la théorie de R. est modulaire, car elle s'ajuste en fonction des compétences encyclopédiques ou culturelles de l'analyste, de ses centres d'intérêt et des niveaux d'analyse visés. Plus qu'une théorie, il s'agit pertinemment d'une praxis stylistique, qui établit une relation définie entre le réel traité et le mouvement littéraire, une praxis attachée à la compréhension dynamique de la production de sens. Elle suppose de ce fait parallèlement une dynamique du sujet interprétant, et donc la prise en compte de sa relation aussi au réel. On aboutit, ainsi que l'envisage Molinié (2010 : 17), à « une stylistique de caractère plutôt socio-culturel, ou sémiosocio-culturel, qui s'attacherait à modéliser des types de lectorat à connecter à des types de littérarité ».

On n'insistera jamais assez, cette exigence contextuelle est pour ainsi dire aux bornes de tout projet stylistique, car elle fixe, suscite et élucide la perception du procédé stylistique, en même temps qu'elle émane d'un complexe d'anticipations et de rétroactions. On voit en tout cas comment l'outillage de R. est conçu pour fondre la structure stylistique à un ensemble de textes issus du même habitus, afin d'en prolonger le rendement et même déterminer la première étape de la démarche stylistique : le repérage des faits. Pas sûr en effet qu'un suffixe aussi anodin que -eur puisse être avalisé, hors contexte, comme stimulus. Quant aux radicaux auxquels est associé le suffixe, il n'est pas certain non plus qu'ils soient, in abastracto, considérés comme contextes stylistiques de chaque idéologème analysé. Le morphème prend forme en une combinaison de rapports qui ne peut plus être simplement linguistique, mais caractéristique d'un style, et même d'un style d'être.

\section{Conclusion :}

In fine, loin d'être immanentiste, la vision riffaterrienne du style se veut comme ressortissant plus au champ littéraire qu'au seul texte. Peut-on alors taxer d'immanentiste une œuvre qui a consacré une part importante de ses problématiques au code-cliché, aux formes conventionnelles, et donc aux groupements stylistiques, et qui, surtout, a fait de l'intertextualité un concept essentiel de son arsenal heuristique ? Un savoir qui mesurait déjà à l'époque combien «l'analyse des styles littéraires collectifs nous apprend davantage sur la littérature que ne saurait le faire l'étude des faits de style individuels » (Schaeffer, 1997 : 17) ? Loin d'être sclérosée, la méthode riffaterrienne détient une géométrie variable à souhait, confinée uniquement aux limites du potentiel encyclopédique du décodeur et à ses visées.

L'on aurait pu explorer d'autres axes linguistiques comme l'appareil énonciatif, ou développer davantage les points communs du dispositif riffaterrien avec l'actualité de la recherche stylistique, notamment son extension à de nouvelles perspectives comme la sociologie de la littérature, ou encore la critique littéraire, qui vient avaliser a posteriori certaines pistes d'analyse. Mais un modeste aspect de la néologie aura suffi, nous l'espérons, à prouver le colossal potentiel transdisciplinaire du style dont R. est l'un des piliers forts, à peser la mesure des convergences significatives qui activent ces ressources, à signaler de nouvelles voies permettant de surmonter certaines critiques qui ont été adressées à la stylistique, enfin, à démontrer ce que la recherche moderne la plus vive continue à lui devoir. A notre avis, la stylistique a tout à gagner, dans ses rapports avec d'autres disciplines, à redorer le blason de l'approche structurale, seule interface épistémologique témoin de la spécificité de la discipline et de la solidité de ses bases.

Si nous nous situons à présent dans l'ensemble du mouvement structuraliste des années 60-70, il est tout aussi clair que la vision de R. se démarque radicalement des autres théories. Tandis qu'il est désormais question chez d'autres de s'inscrire dans quelque anti, post ou néo-structuralisme pour réarticuler le projet, la contextualisation de la stylistique structurale ne nécessite point d'importants réaménagements. Elle a aujourd'hui la capacité de résoudre les nouvelles questions posées aux théories structuralistes, sans renoncer à ce qui la fonde comme discipline. L'heure est effectivement, depuis plusieurs années déjà, au renouvellement de l'incomplet et cependant salutaire structuralisme. En en questionnant les assises épistémologiques, d'aucuns en viennent à souhaiter un ajustement global de la définition même de structure : « mieux vaudrait alors définir une structure comme un réseau autonome de relations où les relations locales sont surdéterminées par les relations globales et dont en outre la stabilité interne peut à 
tout moment être ébranlée par des perturbations externes » (Rastier, 2006 : 99). Pareil souhait était déjà réalisé chez un $\mathrm{R}$. qui, tout en inscrivant substantiellement le déni du seul système linguistique comme composante de l'organicité stylistique, faisait reposer l'essentiel de sa démarche structurale sur le texte dans ses relations à d'autres textes et à ses réceptions. Cette «stabilité interne ébranlée par des perturbations externes » fut donc d'ores et déjà consignée dès la genèse du structuralisme riffaterrien.

Une réserve toutefois : outre le fait que la stylistique structurale se développe à un moment où structuralisme rime avec enfermement, force est de reconnaître que R. n'est pas tout à fait étranger à la vision réductionniste que certains se font de son outillage heuristique. L'élaboration du macrocontexte, surtout, appliqué à un espace de texte de longueur plus ou moins variable, fait apparemment de la matérialité graphique l'horizon extrême pour la saisie du style. Ainsi, même si la question de savoir où et quand commence le contexte stylistique, qui crée les conditions de ressenti pour le stimulus, demeure pertinente, celle de savoir où s'arrête le macrocontexte est laissée en suspens ${ }^{13}$. Car l'amplitude du micro/macrocontexte telle qu'élaborée ne rend pas clairement compte des paliers du pattern stylistique et ses typologies.

Sans prétendre apporter une réponse absolue à la question, nous proposerions, à titre préliminaire, une vision modulaire de l'axe contextuel, en laissant le microcontexte comme tel, mais en disloquant le macrocontexte, quitte à générer une formule du type mésocontexte stylistique. Sans référer au sens strict où Rastier (2004) l'entend, c'est-à-dire vis-à-vis des échelles comprises entre la période et le chapitre, elle restituerait littéralement le sens de la particule grecque méso (au milieu, médian), pour rendre compte du plan intermédiaire entre le microcontexte déclenchant le fait stylistique sur le plan graphique et le macrocontexte, qui est plus tributaire de la mémoire, du savoir. Pleinement doté cette fois-ci du sens de macro, il serait capable de saisir la mesure du double fonctionnement du texte littéraire, dans sa référence non seulement au contenu narré, mais aussi au hors texte, y compris la pratique littéraire elle-même. L'on aboutirait ainsi à une vision des micro et macro contextes comme deux points de départ d'essences différentes (l'un qui crée le stimulus et l'autre par rapport auquel ce stimulus est apprécié et articulé dans les consolidations de sa rentabilité), plutôt que comme deux échelles / mesures de proximité avec le stimulus $^{14}$. Cela permettra surtout, dans la dialectique individuel - collectif - universel, de contribuer à poser / élucider de façon pertinente le problème du jeu de distinction entre ceux des procédés stylistiques dont la reconnaissance est conditionnée par les propriétés du contexte et ceux que ces propriétés viennent éclairer de façon contingente.

\section{Eléments bibliographiques}

Bourdieu, P. (1980). Le Sens pratique. Paris : Editions de Minuit.

Bourdieu, P. (1994). Raisons pratiques. Sur la théorie de l'action. Paris : Le Seuil.

Cressot, M. et James, L. (1988). Le style et ses techniques. Paris : PUF.

Genette, G. (1966). « Structuralisme et critique littéraire ». In Figures I. Paris : Le Seuil - coll. Points Essais, pp. 155170.

Goodman, N. (1978). «Le statut du style ». In Manières de faire des mondes. Trad. fr. de Popelard, M.-D. (2006). Paris : Gallimard - coll. Folio-Essais, pp. 44-66.

Hardy, A. (1969). « Théorie et méthode stylistiques de Riffaterre ». Langue française, ${ }^{\circ}$ 3, pp. 90-96.

Hopkins, J. (2005). «La théorie sémiotique littéraire de Michael Riffaterre: matrice, intertexte et interprétant ». Cahiers de Narratologie, $\mathrm{n}^{\circ} 12$ [en ligne]. URL : http://narratologie.revues.org/37.

Thomas, J.-J. (2002). «Riffaterre et la lecture herméneutique de la poésie française ». The Romanic Review, vol. 93, $n^{\circ} .1-2$, pp. $15-30$.

Kouassi, G. (2007). Le Phénomène de l'appropriation linguistique et esthétique en littérature africaine de langue française. Le cas des écrivains ivoiriens : Dadié, Kourouma, Adiaffi. Paris : Publibook.

Kristeva, J. (2002). «Nous deux or a (hi)story of intertextuality ». The Romanic review, vol. 93, n 1-2, pp. 7-13. 
Maingueneau, D. et Philippe, G. (2002). «Les conditions d'exercice du discours littéraire ». In Roulet, E. et Burger, M. (éds). Les Modèles du discours au défi d'un «dialogue romanesque ». Nancy: Presses Universitaires de Nancy, pp. 351-378

Molinié, G. (2010). «Pratique et herméneutique ». In Bougault, L. et Wulf, J. (éds). Stylistiques ?. Rennes : Presses Universitaires de Rennes, pp. 15-19.

Philippe, G. (2005). "Traitement stylistique et traitement idiolectal des singularités langagières ». Cahiers de praxématique : L'idiolecte, $\mathrm{n}^{\circ} 44$, pp. 77-92.

Piat, J. (2007). «Vers une stylistique des imaginaires langagiers ». Corpus : Corpus et stylistique, n 5, pp. 2-16.

Rabaté, J.-M. (2002). «Michael Riffaterre and the unfinished project of structuralism ». The Romanic Review, vol. 93, no. 1-2, pp. 31-44.

Rastier, F. (1998). «Le problème épistémologique du contexte et le statut de l'interprétation dans les sciences du langage ». Langages, $n^{\circ} 129$, pp. 97-111.

Rastier, F. (2004). «Enjeux épistémologiques de la linguistique de corpus », Texto !. [en ligne]. URL: http://www.revue-texto.net/Inedits/Rastier/Rastier_Enjeux.html.

Rastier, F. (2006). « La structure en question ». Janus : Quaderni del Circolo Glossematico, $\mathrm{n}^{\circ}$ 6, pp. 93-104.

Riffaterre, M. (1971). Essais de stylistique structurale. Paris : Flammarion.

Riffaterre, M. (1974). « Paragram and Significance ». Semiotext(e), n 1-2, pp. 72-87.

Riffaterre, M., (1979). « Sémiotique intertextuelle : l'interprétant ». Revue d'esthétique, $\mathrm{n}^{\circ}$ 1-2, pp. 128-150.

Riffaterre, M. (1980). « La trace de l'intertexte ». La pensée, n 215, pp. 4-18.

Riffaterre, M. (1981). «L'intertexte inconnu ». Littérature, n41, pp. 4-7.

Riffaterre, M. (1994). «L'inscription du sujet». In Molinié, G. et Cahné, P. (éds). Qu'est-ce que le style ?. Paris : P.U.F., pp. 283-312.

Schaeffer, J.-M. (1997). « La stylistique littéraire et son objet ». Littérature, n 105, pp. 14-23.

Vandendorpe, C. (1991). «Contexte, compréhension et littérarité ». RS/SI, vol. 11, n 1, pp. 9-25.

Vessah Ngou, D. (2014). « Le paradoxe de la poule et de l'œuf. Essai sur les enjeux entre la création et la critique sur le champ littéraire africain ». Ecritures, $\mathrm{n}^{\circ} 12$, pp. 115-133.

\footnotetext{
${ }^{1}$ Voir par exemple l'article de Jean-Michel Rabaté (2002) « Riffaterre and the unfinished project of structuralism ».

${ }^{2}$ L'on lira avec profit, entre autres et outre la contribution de Jean-Michel Rabaté mentionnée supra, les travaux d'Alain Hardy, de John Hopkins, de Jean-Jacques Thomas, de Julia Kristeva, ou encore l'ensemble de l'hommage rendu à Riffaterre dans The Romanic review (2002) de Columbia University.

${ }^{3}$ Il paraît évident que cette étroitesse est due en majeure partie au fait que l'essence du dispositif théorique et des travaux de R., comme on le sait, est centrée de manière générale sur le poème, même si le théoricien s'en défend, comme l'indique la note de la traduction à la page 27 des Essais : « d'un commun accord, l'auteur et le traducteur ont décidé $[. .$.$] de ne pas s'en tenir à une traduction littérale [des] termes, eu égard à certaines confusions auxquelles ils$ ont donné lieu [...]; quant à poem, parfois nous garderons "poème » qu'il faudra alors prendre dans son sens étymologique, parfois, en particulier s'il y a risque de confusion, nous lui substituerons «texte ", notion qui est aujourd'hui au premier plan des recherches stylistiques ».

${ }^{4}$ R. insiste beaucoup sur le pôle de l'analyste, qui établit les étalons au profit d'un usage subséquent. Mais il prend peu la mesure des multiples influences que ces jalons posés par le stylisticien peuvent avoir sur le pôle de la création, qui en est parfois à l'affût pour élaborer une part considérable de ses stratégies. C'est un détail qui n'est pas à négliger dans le décryptage des idées littéraires et des formes par lesquelles elles se manifestent, surtout en ce qui concerne certaines formations esthétiques qui, loin d'être des générations spontanées, se nourrissent essentiellement de la sève des apports des critiques, bien plus que de celle des lecteurs ordinaires. Nous avons développé certaines de ces incidences dans un article récent intitulé « Le paradoxe de la poule la poule et de l'œuf. Essai sur les enjeux entre la création et la critique sur le champ littéraire africain ».
} 


\begin{abstract}
${ }^{5}$ Si le patron stylistique fait montre d'une élaboration théorique satisfaisante envers la perception des imaginaires langagiers proposés comme étalons, ce qu'il a en commun avec le pattern stylistique de $\mathrm{R}$. ne peut échapper à l'attention. Ce n'est d'ailleurs pas un hasard si certains traducteurs de R., à l'instar de Hardy (1969: 91), restituent l'expression pattern par patron lorsqu'ils convoquent le stylisticien : «Le contexte est conçu " comme un patron (pattern) rompu par un élément imprévisible ». Quoi qu'il en soit, les deux termes sont polysémiques et renvoient à : (i) la manière spécifique dont un fait est survenu ou la façon dont il a été soit réalisé, soit organisé ; (ii) toute forme plastique dont l'occurrence est répétée; (iii) quelque chose qui est montré pour servir de référence, de modèle à reproduire ; (iv) un standard qui commande des opérations futures. Mais il faut reconnaître que, nonobstant son stade d'aménagement assez avancé et articulé au métalangage actuel, le terme patron a non seulement l'avantage d'être français mais aussi celui de ressortir avec pertinence le sens de chef qui dirige une opération. De fait, il se charge de fixer des repères afin de porter à l'attention des phénomènes inspirateurs. Mais cela ne signifie en rien que ce n'est pas le même esprit directeur dont était déjà doté le pattern de R. (1971), qui en parle tantôt comme phénomène qui commande l'attention du lecteur (p. 39), tantôt comme procédé de contrôle du décodage (p. 62), ou encore comme préparateur et générateur du contraste (p. 87), ou même comme jalon des points du discours où les réactions du lecteurs présupposent l'existence de stimuli (p. 111). Bref, les actualisations sont imposées par les patterns (p. 38).
\end{abstract}

${ }^{6}$ Précisons aussitôt à quoi renvoient les abréviations (marquées entre parenthèses), avec les références des romans :

Hampâté Bâ, A. (1994). Oui mon commandant! (OM.C.). Paris : Actes du Sud.

Kourouma, A. (1968). Les Soleils des indépendances (S.I.). Paris, Montréal : Presses de l'Université de Montréal.

Kourouma, A. (1990). Monnè, outrages et défis (M.O.D.). Paris : Le Seuil.

Kourouma, A. (2000). Allah n'est pas obligé (A.P.O.). Paris : Le Seuil.

Labou Tansi, S. (1979). La Vie et demie (L.V.D.). Paris : Le Seuil.

Labou Tansi, S. (1983). L'Anté-peuple (A.P.). Paris : Le Seuil.

Labou Tansi, S. (1985). Les Sept solitudes de Lorsa Lopez (S.S.L.L.). Paris : Le Seuil.

Labou Tansi, S. (1988). Les Yeux du volcan (Y.V.). Paris : Le Seuil.

Labou Tansi, S. (1995). Le Commencement des douleurs (C.D.). Paris : Le Seuil.

Miano, L. (2006). Contours du jour qui vient (C.J.V.). Paris : Plon.

Monénembo, T. (2000). L'Aîné des orphelins (A.O.). Paris : Le Seuil.

Monénembo, T. (2008). Le Roi de Kahel (R.K.). Paris : Le Seuil.

Ousmane, S. (1973). Xala (X.). Paris : Présence africaine.

${ }^{7}$ Avant d'aller plus loin dans nos analyses, une mise au point s'impose : l'on pourrait objecter que certaines de ces formes répertoriées sont présentes dans d'autres sociétés, ou qu'elles ne sauraient faire l'objet de quelque intérêt puisqu'elles sont déjà répertoriées dans le lexique (pleureuses, tombeurs, etc.). Une telle considération, qui revient à se situer par rapport à la norme linguistique ou à définir le procédé stylistique en termes de distribution et de fréquences, repose avec acuité une question ardue pour l'essence du style : le propre est-il de l'ordre de l'exclusif? Rappelons les facteurs qui invalident de tels repères universalistes : premièrement, le fait pour une unité linguistique d'être neutre indépendamment des autres ne signifie en rien qu'elle le restera une fois appréciée par rapport à l'idiolecte d'un auteur, ou à quelque structure socio-historico-littéraire précise, car ce qui importe, ce n'est pas la connaissance de la langue comme système de signes virtuels, mais plutôt celle d'une expérience de celle-ci; secondement, le code écrit est susceptible de jouer le rôle de catalyseur en faveur du renouvellement d'un élément linguistique du langage oral, au moins en vertu des relations qui l'organisent dans son nouvel environnement textuel ; troisièmement, il ne faudrait pas oublier que la transcription d'unités linguistiques courantes dans des séquences littéraires les rend potentiellement calculées, et donc dignes d'intérêt. Last but not least, c'est toute la vitalité de la stylistique qui pourrait être mise à mal à vouloir dégager des valeurs stylistiques permanentes. Le discours devrait s'imposer prioritairement comme signe plutôt que comme forme ou contenu. Le stylisticien se doit de garder en vue la prééminence du fait littéraire sur le fait de langue ou même le fait d'auteur : " tout élément du discours, même le plus ordinaire, peut devenir un fait de style et entrer dans la composition d'une structure littéraire. » («Vers la définition linguistique du style » in R., $1971: 120)$. 


\footnotetext{
${ }^{8}$ Nous avons choisi le Shu pamom parce que c'est la langue que nous maîtrisons le plus, mais le même principe régit plusieurs autres parlers.

${ }^{9}$ Cela va sans dire que même si nous avons symboliquement opté pour le seul suffixe -eur, d'autres suffixes ou procédés (dérivation impropre par exemple : les assis, les accouchées, les arrivants, etc.) liés à l'attitude ou à la profession peuvent conduire à des interprétations et à un rendement similaires. A ce titre, le fait que le morphème ne surgisse qu'à la faveur de sa logique fonctionnelle ou professionnelle est significatif de la prééminence liée à la tendance sémantique plutôt que morphologique. En effet, sans être absent du corpus, il ne jouit absolument pas de la même proportion au moment de signifier la qualité, le caractère ou la relation, comme dans fadeur, tiédeur, etc.

${ }^{10}$ Il est vrai qu'il faut situer ce déni dans la perspective idiolecte = style, qui enferme la vision du style dans des concepts problématiques tels que le choix, ou la personnalité d'auteur, selon la critique génétique.

${ }^{11}$ Relativement à un approfondissement des enjeux stylistiques posés par l'opposition idiolecte / sociolecte, l'on lira avec profit l'article de Philippe (2005).

12 Concernant particulièrement le champ littéraire africain, ces écueils n'exposent pas qu'à des risques de sousinterprétation, où l'élucidation resterait en deçà d'un seuil de pertinence rationnel, mais ils touchent aussi à certaines mésinterprétations et surinterprétations qui déforment l'analyse littéraire par des assimilations culturelles non ajustées. Voilà pourquoi, sans préconiser un ésotérisme de la démarche et sans non plus vouloir réduire les écarts stylistiques des auteurs à de simples différences culturelles, l'on est favorable à une approche des textes qui contrôlerait leur soubassement culturel, selon les perspectives d'analyse. Nous développons plus profondément un tel parti pris dans notre article « Chassez l'ethnostylistique par la porte, elle rentrera par la fenêtre » (à paraître).

${ }^{13}$ En définissant le macrocontexte dans « Le contexte stylistique » (p. 81), R. dialectisait : « où s'arrête le contexte ? cela est connu par définition; où commence-t-il ? Le problème est plus difficile à résoudre. » Et pourtant il semble que la vraie question est : « où s'arrête le contexte ? » Concentré sur l'arrière-plan qui nourrit les réactions du lecteur, ou qui est à l'origine de son édification sur certains faits contrastants, R. néglige le caractère fluctuant du contexte dans son extrémité supérieure.

${ }^{14} \mathrm{C}$ 'est l'économie des substrats textuel et intertextuel sous le macrocontexte riffaterrien qui fait que le mésocontexte stylistique ne saurait renvoyer seulement au palier mésotextuel de Rastier, mais devrait englober, en plus, le palier macrotextuel (texte complet dont péritexte et paratexte). Soit schématiquement : mésocontexte stylistique $=$ plans mésotextuel + macrotextuel de Rastier. Bien évidemment, le plan intertextuel de Rastier ne rejoint pas non plus la structure intertextuelle de R.: tandis que le premier, confiné au corpus, est d'ores et déjà le produit d'une construction à dessein pour la validité des résultats, le second, quant à lui, fait l'objet de prospections et de rétrospections ; il est cognitif, abstrait, difficilement saisissable dans ses limites. D'où l'interrogation de R. sur « où commence le contexte ». Il va sans dire que ces divergences relèvent surtout de la substance différentielle du texte et du contexte. Quoique les deux soient parentés, notamment dans les problèmes que posent leurs limites respectives, le contexte recèle fondamentalement une proportion fluide, par rapport au texte, qui se saisit dans sa matérialité, qu'elle soit graphique ou phonique.
} 